\title{
(Pre-)sheaves of Ring Spectra over the Moduli Stack of Formal Group Laws*
}

\author{
Paul G. Goerss ${ }^{\dagger}$
}

\begin{abstract}
In the first part of this article, I will state a realization problem for diagrams of structured ring spectra, and in the second, I will discuss the moduli space which parametrizes the problem. While some of what I say is quite general, the ring spectra I have in mind will arise from the chromatic point of view, which uses the geometry of formal groups to organize stable homotopy theory. Thus, a subsidiary aim here is to reemphasize this connection between algebraic geometry and homotopy theory.
\end{abstract}

\section{Contents}

1 The Realization Problem $\quad 2$

1.1 An extremely short introduction to stacks . . . . . . . . . . . . . . . . . 2

1.2 Some algebraic properties of stacks . . . . . . . . . . . . . . . . . 5

1.3 Sheaves and cohomology . . . . . . . . . . . . . . . . . . . . 7

1.4 The realization problem . . . . . . . . . . . . . . . . . . . . . . 10

2 Moduli Spaces and Obstruction Theory $r$

2.1 The Dwyer-Kan moduli space . . . . . . . . . . . . . . . . . . . . . . 13

2.2 André-Quillen cohomology . . . . . . . . . . . . . . . . . . . . . . . . 14

2.3 The Dwyer-Kan-Stover decomposition of $B \mathcal{R}(A) \ldots \ldots \ldots$

2.4 Commutative $v s$ non-commutative: the role of the cotangent complex . . . . . . 17

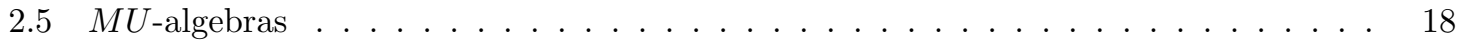

2.6 Hopkins-Miller solves a moduli problem . . . . . . . . . . . . . . . . . 20

Before even starting, I should say that all of the first section amounts to a meditation on work of Mike Hopkins and Haynes Miller. There are people who know more about various aspects of this area than I do - in addition to Hopkins and Miller, of course. Matt Ando, Charles Rezk, and Neil

\footnotetext{
* This note is an expanded version of two talks given during the NATO Advanced Study Institute "Axiomatic and Enriched Homotopy Theory" held at the Isaac Newton Institute for Mathematical Sciences, Cambridge, UK, 9-20 September 2002.

†The author was partially supported by the National Science Foundation.
} 
Strickland come to mind immediately, for example, and any interested reader would do well to look at [2], [3], [20], [21] [34], [38], and [39]. The table in section 2 of the paper by Hopkins and Gross [21] indicates just how far behind the times I am. The second section owes much to Bill Dwyer. The mistakes, obfuscations, over-simplifications, and sweeping generalizations are mine.

I should also say that, especially in the material on stacks, I have made no attempt to display all the details of a rather intricate subject.

\section{The Realization Problem}

The basic question is this: when can certain interesting sheaves of rings be lifted to presheaves of $E_{\infty}$-ring spectra? The spectra and cohomology theories that arise in the chromatic point of view of stable homotopy theory tend to be parametrized by stacks of a particular type; thus, to make the questions concrete, a certain amount of background is required.

\subsection{An extremely short introduction to stacks}

Stacks were introduced by Deligne, Mumford, Artin, and others to parametrize problems in algebraic geometry where the presence of automorphisms prevented representability by a scheme or an algebraic space. An early example was the moduli stack $\mathcal{M}_{g}$ which classified curves of a given genus [9], but the paper of Artin [4] was explicit about this point of view. The use of these ideas has expanded to the point where there is now a whole cottage industry on stacks, and they are supposed to be part of our basic tool kit. The standard book reference on this subject is [27] and a concise introduction to the algebraic geometry point of view is in the appendix to [41]. A relative recent introductory paper also exists; see [16]. The basic idea is rather simple (see the example immediately following), but to make use of this idea we must impose conditions, and this leads into technicalities I will try to not belabor.

A stack is a sheaf of groupoids that satisfy effective descent, which is a gluing condition for isomorphisms explicated by the next example.

Example 1.1. Let $X$ be a topological space. Then to each open subset $U \subseteq X$ we assign the groupoid $\operatorname{Bund}_{n}(U)$ with objects the real $n$-plane bundles over $U$ and the morphisms bundle isomorphisms over $U{ }^{1}$ As $U$ varies, $\operatorname{Bund}_{n}$ is a sheaf of groupoids, but more is true. Suppose $\left\{V_{i}\right\}$ is an open cover of $U$ and we have bundles $\xi_{i}$ over $V_{i}$ and bundle isomorphisms

$$
\phi_{i j}:\left.\left.\xi_{i}\right|_{V_{i} \cap V_{j}} \stackrel{\cong}{\longrightarrow} \xi_{j}\right|_{V_{i} \cap V_{j}}
$$

over $V_{i} \cap V_{j}$. If these isomorphism satisfy the obvious cocycle conditions, then they glue: there is a bundle $\xi$ over $U$ and isomorphisms

$$
\phi_{i}:\left.\xi\right|_{V_{i}} \stackrel{\cong}{\longrightarrow} \xi_{i}
$$

over $V_{i}$. Abstracted, this is the effective descent condition.

While this example is familiar to algebraic topologists - it's why we need $K$-theory in non-zero degrees - it is not a very good one for a number of reasons. One defect is that the assignment

\footnotetext{
${ }^{1}$ To make the objects of this groupoid a set, I should take bundles equipped with a linear embedding to $X \times \mathcal{U}$, where $\mathcal{U}$ is some fixed real inner product space of countable infinite dimension.
} 
$U \mapsto \operatorname{Bund}_{n}(U)$ is not really a functor: if

$$
U_{1} \stackrel{f}{\longrightarrow} U_{2} \stackrel{g}{\longrightarrow} U_{3}
$$

is a sequence of composable maps and $\xi$ is a bundle over $U_{3}$, then $(g f)^{*} \xi$ and $f^{*} g^{*} \xi$ are only isomorphic, not equal. This is not really a serious problem, and can be handled by the device of using "categories fibered in groupoids". See [27] or [22]. (The latter source is explicit about the various equivalences of categories needed.) More seriously, this example doesn't evidently support the rich algebraic structures of algebraic geometry.

Before going on to the next example, note that stacks form a full subcategory of the category of presheaves of groupoids, and the inclusion functor from stacks to presheaves of groupoids has a left adjoint. We could call this "stackification", but this is not a pleasant sounding word, so I will call it the associated stack functor. At this point it is worth mentioning that there is a model category structure on presheaves of groupoids for which stacks form the fibrant objects. See [22] and [26].

I am now going to begin using some of the language of algebraic geometry, but mostly in the relatively abstract sense of, say, the first part of Demazure and Gabriel [10]. For example, if $R$ is a commutative ring, then $\operatorname{Spec}(R)$ means the representable functor $\operatorname{Hom}(R,-)$ on the category of commutative rings. Of course, more geometric descriptions of $\operatorname{Spec}(R)$ can be recovered from this one. All rings in this note are commutative, except where I explicitly stipulate otherwise.

Example 1.2 (Hopf algebroids). Let $(A, \Gamma)$ be a Hopf algebroid. By definition, for each ring $R$, the sets $\operatorname{Hom}(A, R)$ and $\operatorname{Hom}(\Gamma, R)$ of ring homomorphisms form, respectively, the objects and morphisms of a groupoid which I will write $G_{\Gamma}(R)$. I will always assume that the left unit $\eta_{L}: A \rightarrow \Gamma$ is a flat map.

The assignment

$$
\operatorname{Spec}(R) \mapsto G_{\Gamma}(R)
$$

is a sheaf in any of the algebraic topologies for which the representable presheaves are sheaves: Zariski, étale, flat, etc. ${ }^{2}$ For example, if $R \rightarrow S$ is a faithfully flat extension (so that $\operatorname{Spec}(S) \rightarrow$ $\operatorname{Spec}(R)$ is a cover in the flat topology) then

$$
G_{\Gamma}(R) \longrightarrow G_{\Gamma}(S) \Longrightarrow G_{\Gamma}\left(S \otimes_{R} S\right)
$$

is an equalizer diagram of groupoids. To see this, note that the after applying the functor $(-) \otimes_{R} S$, the diagram of rings

$$
R \longrightarrow S \Longrightarrow S \otimes_{R} S
$$

becomes a split equalizer diagram, and this is enough by faithfully flat descent. However, $G_{\Gamma}$ is almost never a stack. Let us write $\mathcal{M}(A, \Gamma)$ for the stack (in the flat topology) obtained by applying the associated stack functor.

As a warning, it might be worth mentioning that more sophisticated topologies could be used here and in the definition of sheaves in section 1.3 below - more than that, this might be desirable. It is a drawback that the flat topology doesn't have enough points, for example. See Remark 1.13 for more on topologies. But see Example 1.9 for a reason to start with the flat topology.

\footnotetext{
${ }^{2}$ And, as a result, I could expand the domain of definition of this groupoid to schemes if I needed to.
} 
Example 1.3. A degenerate example of the previous example is the case when $A=\Gamma=R$ for some commutative ring $R$. Then $\mathcal{M}(A, \Gamma)=\operatorname{Spec}(R)$ is already a stack - as the only isomorphisms are the identity, which makes the effective descent condition trivial. Furthermore, upon examining the associated stack functor, we note that a morphism of stacks ${ }^{3}$

$$
x: \operatorname{Spec}(R) \longrightarrow \mathcal{M}(A, \Gamma)
$$

corresponds to an equivalence class of pairs $\left(f: A \rightarrow S, \phi: \Gamma \rightarrow S \otimes_{R} S\right)$ where $R \rightarrow S$ is a faithfully flat extension and $\phi$ is an isomorphism between the two induced elements $A \rightarrow S \otimes_{R} S$. Of course, $\phi$ must satisfy a cocycle condition, and the two pairs $(f, \phi)$ and $\left(f^{\prime}, \phi^{\prime}\right)$ induce the same map $x$ if they have a common refinement. In this sense, $\mathcal{M}(A, \Gamma)$ is a moduli or classifying object for elements of the groupoid $G_{\Gamma}$.

Example 1.4 (Formal group laws). The bedrock example of this note is the moduli stack of formal group laws. Let

$$
L=\mathbb{Z}\left[a_{1}, a_{2}, \ldots\right]
$$

be the (ungraded) Lazard ring. Then $\operatorname{Hom}(L, R)$ is naturally isomorphic to the set of formal group laws over $R$. Also let

$$
W=L\left[b_{0}^{ \pm 1}, b_{1}, b_{2}, . .\right]
$$

classify (non-strict) isomorphisms between formal group laws. Let us write $\mathcal{M}_{\mathcal{F G} \mathcal{L}}$ for the resulting stack. Then, by the last example, morphisms

$$
\operatorname{Spec}(R) \rightarrow \mathcal{M}_{\mathcal{F} \mathcal{G L}}
$$

classify equivalence classes of formal groups laws over faithfully flat extensions of $R .^{4}$

Example 1.5 (Elliptic curves). The moduli stack $\mathcal{M}_{\text {ell }}$ of (Weierstrass) elliptic curves can be written in the form $\mathcal{M}(A, \Gamma)$ for a suitable Hopf algebroid $(A, \Gamma)$. Indeed, every elliptic curve over $R$ has unique expression as the projective completion of the affine curve

$$
y^{2}+a_{1} x y+a_{3} y=x^{3}+a_{2} x^{2}+a_{4} x+a_{6}
$$

with $a_{i} \in R$. This is the Weierstrass form of the curve. Writing down such an equation does more than uniquely specify the curve. It also uniquely specifies a choice of local coordinate near the identity of the curve (and hence a coordinate of the associated formal group law) "modulo degree 5". Automorphisms of this situation are the projective transformations determined by formulas

$$
x \mapsto \lambda^{2} x+r \quad y \mapsto \lambda^{3}+s \lambda^{2}+t
$$

with $r, s, t \in R$ and $\lambda \in R^{\times}$. Thus

$$
A=\mathbb{Z}\left[a_{1}, a_{2}, a_{3}, a_{4}, a_{6}\right]
$$

and $\Gamma=A\left[r, s, t, \lambda^{ \pm 1}\right]$

There is a morphism of stacks $\mathcal{M}_{\text {ell }} \rightarrow \mathcal{M}_{\mathcal{F G} \mathcal{L}}$ which assigns to each elliptic curve the formal group obtained by formal completion at the identity. All of this can be recovered from [34]; see [20] for the wider implications.

\footnotetext{
${ }^{3}$ I really mean 1-morphisms of stacks, to use the language of 2-categories.

${ }^{4}$ We could hope for a more concrete description of these equivalence classes, but I don't know one. If we work with formal groups, rather than formal group laws (the groupoids, and hence the stacks, are equivalent), such a description is possible, provided we allow the invariant differentials (see 1.15) to be an invertible module, rather than simply a free module of rank 1 .
} 
Example 1.6. A commuting (or 2-commuting) diagram of stacks, written as

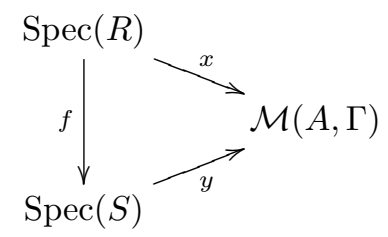

is a pair $(f, \phi)$ where $f: S \rightarrow R$ is a ring homomorphism and $\phi$ is an isomorphism $\phi: x \rightarrow f^{*} y$. Thus we only require commutativity up to isomorphism and the isomorphism is part of the data. To make this more concrete, consider the case when $\mathcal{M}(A, \Gamma)=\mathcal{M}_{\mathcal{F} \mathcal{G} \mathcal{L}}$. Then $x$ and $y$ can be represented, respectively, by formal group laws $G$ and $H$ over some faithfully flat extensions, and we specify an isomorphism $f^{*} H \cong G$, again, perhaps, after further extension.

We write $(f, \phi):(\operatorname{Spec}(R), x) \rightarrow(\operatorname{Spec}(S), y)$ for the 2-commuting diagram.

\subsection{Some algebraic properties of stacks}

Here I impose the bare necessity of what is needed from the sheaf-theoretic part of algebraic geometry to make a statement of the realization problem. The basic intuition with stacks is that they should have most of the rich structure that makes schemes such an excellent object of study. This includes a structure sheaf, quasi-coherent sheaves of modules, invertible sheaves, and so on. This kind of structure is not available for Example 1.1, but it is true of all the others.

First, a construction. Given a diagram<smiles>[AlH2][AlH]Cl</smiles>

of groupoids the homotopy pull-back $G_{1} \overrightarrow{\times}_{H} G_{2}$ is the groupoid with objects consisting of triples $(x, y, \phi)$ with $x$ and $y$ objects in $G_{1}$ and $G_{2}$ respectively and $\phi: f(x) \rightarrow g(y)$ is an isomorphism in $H$. A morphism $(x, y, \phi) \rightarrow\left(x^{\prime}, y^{\prime}, \phi^{\prime}\right)$ is a pair of isomorphisms $x \rightarrow x^{\prime}$ and $y \rightarrow y^{\prime}$ so that the evident square in $H$ commutes. This name "homotopy pull-back" is non-standard terminology, but appropriate: after taking nerves, we get a homotopy pull-back of spaces. Most authors would call this the 2-category pull-back.

The construction of such homotopy pull-backs evidently globalizes to presheaves of groupoids. We will say that a morphism

$$
\mathcal{M} \longrightarrow \mathcal{N}
$$

of stacks is representable if whenever we form the homotopy pull-back

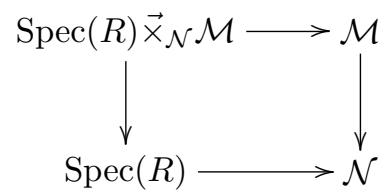


then $\operatorname{Spec}(R) \overrightarrow{\times}_{\mathcal{N}} \mathcal{M}$ is discrete; that is, there is a commutative ring $S$ and an equivalence of groupoid sheaves $\operatorname{Spec}(S) \simeq \operatorname{Spec}(R) \overrightarrow{\times}_{\mathcal{N}} \mathcal{M} .^{5}$

Once we have a representable morphism of stacks, we can extend many of the definitions of algebraic geometry to stacks. For example, if $P$ is any property of morphisms of schemes stable under base change, then one can say a representable morphism $\mathcal{M} \rightarrow \mathcal{N}$ of stacks has property $P$ if for all morphisms $\operatorname{Spec}(R) \rightarrow \mathcal{N}$, the resulting map of schemes

$$
\operatorname{Spec}(S) \simeq \operatorname{Spec}(R) \overrightarrow{\times}_{\mathcal{N}} \mathcal{M} \rightarrow \operatorname{Spec}(R)
$$

has property $P$.

Example 1.7. Let $(A, \Gamma)$ be a Hopf algebroid. Then $y: \operatorname{Spec}(S) \rightarrow \mathcal{M}(A, \Gamma)=\mathcal{M}$ is representable. To see this, first suppose that $y: \operatorname{Spec}(S) \rightarrow \mathcal{M}$ and $x: \operatorname{Spec}(R) \rightarrow \mathcal{M}$ are actually given by maps $A \rightarrow S$ and $A \rightarrow R$. Then

$$
\operatorname{Spec}(R) \overrightarrow{\times}_{\mathcal{M}} \operatorname{Spec}(S) \simeq \operatorname{Spec}\left(R \otimes_{A} \Gamma \otimes_{A} S\right) .
$$

More generally, for any map $x: \operatorname{Spec}(R) \rightarrow \mathcal{M}$ there is a faithfully flat extension $i: R \rightarrow R^{\prime}$ so that

$$
i^{*} x: \operatorname{Spec}\left(R^{\prime}\right) \longrightarrow \mathcal{M}
$$

is represented by a map $A \rightarrow R^{\prime}$. Do the same for $y: \operatorname{Spec}(S) \rightarrow \mathcal{M}$ and then use faithfully flat descent to get that $\operatorname{Spec}(R) \overrightarrow{\times}_{\mathcal{M}} \operatorname{Spec}(S)$ is discrete.

Note that, in particular, the map $\operatorname{Spec}(A) \rightarrow \mathcal{M}$ classifying the universal element is surjective and flat, because we assumed that $\eta_{L}: A \rightarrow \Gamma$ is flat.

Example 1.8 (A Čech complex). Here is another observation I learned from Mike Hopkins. Restricting attention to stacks arising from Hopf algebroids is not as confining as one might initially think. Let us call a stack $\mathcal{M}$ algebraic if we can choose a representable, flat, and surjective map

$$
\operatorname{Spec}(A) \rightarrow \mathcal{M}
$$

Having chosen such a cover, let

$$
\operatorname{Spec}(\Gamma) \simeq \operatorname{Spec}(A) \overrightarrow{\times}_{\mathcal{M}} \operatorname{Spec}(A) .
$$

Then, writing $U=\operatorname{Spec}(A)$, we have a Čech resolution

$$
\cdots U \overrightarrow{\times}_{\mathcal{M}} U \overrightarrow{\times}_{\mathcal{M}} U \rightleftarrows U \overrightarrow{\times}_{\mathcal{M}} U \rightleftarrows U \longrightarrow \mathcal{M} \text {. }
$$

This can be rewritten as

$$
\cdots \operatorname{Spec}\left(\Gamma \otimes_{A} \Gamma\right) \underset{⿱}{\rightleftarrows} \operatorname{Spec}(\Gamma) \rightleftarrows \operatorname{Spec}(A) \longrightarrow \mathcal{M}
$$

and, from this, one reads off the structure of a Hopf algebroid on the pair $(A, \Gamma)$. Furthermore, there is a morphism $\mathcal{M}(A, \Gamma) \rightarrow \mathcal{M}$ which is an equivalence of stacks. The reader who has got this far will recognize that Equation 1.1 is Spec of the cobar complex and will suspect that we may have begun to set up an equivalence of categories between Hopf algebroids and certain types of "rigidified" stacks - that is, ones with a chosen affine cover, as above.

\footnotetext{
${ }^{5}$ Meaning we get a weak equivalence of simplicial presheaves by applying the nerve functor to the morphism of presheaves of groupoids. One should loosen the notion of discrete to specify only that $\operatorname{Spec}(R) \vec{x}_{\mathcal{N}} \mathcal{M}$ is equivalent to a scheme. Even when considering various stacks associated to formal groups and formal group laws, open subschemes of affine schemes arise from this construction - and these need not be affine. However, let's keep it simple here.
} 
Example 1.9. It is a result of Hopkins and Miller that $\operatorname{Spec}(R) \rightarrow \mathcal{M}_{\mathcal{F G} \mathcal{L}}$ is flat if and only if the resulting formal group law satisfies a version of Landweber exactness. See the notes by Miller [30].

Example 1.10. It is another result of Hopkins and Miller that $\mathcal{M}_{\text {ell }} \rightarrow \mathcal{M}_{\mathcal{F G} \mathcal{L}}$ is representable. It is not quite flat, because of the presence of certain singular elliptic curves, especially the cusp $y^{2}=x^{3}$. If $\mathcal{M}_{\text {ell }}^{+} \subseteq \mathcal{M}_{\text {ell }}$ is the open substack obtained by avoiding these curves, then the restricted map

$$
\mathcal{M}_{\text {ell }}^{+} \longrightarrow \mathcal{M}_{\mathcal{F G \mathcal { L }}}
$$

is flat.

There might be a simple criterion for when a morphism of Hopf algebroids induces a representable morphism of stacks, but I don't know one and the subtleties of this example make me wonder.

\subsection{Sheaves and cohomology}

We are interested in sheaves of modules on an algebraic stack $\mathcal{M}$. Define a category Flat/ $\mathcal{M}$ to have objects the flat maps

$$
\operatorname{Spec}(R) \longrightarrow \mathcal{M}
$$

and morphisms the 2-commuting triangles. Then define a cover of an object $\operatorname{Spec}(R) \rightarrow \mathcal{M}$ to be a faithfully flat map $\operatorname{Spec}(S) \rightarrow \operatorname{Spec}(R)$. A sheaf, then, is a contravariant functor from Flat/M to sets which yields the expected equalizer diagram for all covers. See Remark 1.13 for other possible topologies.

Example 1.11 (The structure sheaf). The structure sheaf $\mathcal{O}=\mathcal{O}_{\mathcal{M}}$ on $\mathcal{M}$ is the functor

$$
\mathcal{O}(\operatorname{Spec}(R) \rightarrow \mathcal{M})=R
$$

This is a sheaf of rings.

Example 1.12 (Quasi-coherent sheaves). For an affine scheme $\mathcal{M}=\operatorname{Spec}(R)$, the category of quasi-coherent $\mathcal{O}_{\mathcal{M}}$-module sheaves is equivalent to the category $R$-modules. If $\mathcal{M}=\mathcal{M}(A, \Gamma)$, the category of quasi-coherent $\mathcal{O}_{\mathcal{M}}$-modules is equivalent to the category of $(A, \Gamma)$-comodules. Thus the structure sheaf $\mathcal{O}_{\mathcal{M}}$, while simple to define, encodes a great deal of information.

First note that to define a sheaf, we may restrict attention to those objects $x: \operatorname{Spec}(R) \rightarrow \mathcal{M}$ in Flat $/ \mathcal{M}$ so that $x$ is actually represented by a map $A \rightarrow R$. This is because every object has a $\operatorname{cover} \operatorname{Spec}(S) \rightarrow \operatorname{Spec}(R)$ so that the composite

$$
\operatorname{Spec}(S) \longrightarrow \operatorname{Spec}(R) \stackrel{x}{\longrightarrow} \mathcal{M}
$$

can be so represented. Having defined the sheaf on $\operatorname{Spec}(S) \rightarrow \mathcal{M}$, the sheaf condition ensures its definition on $x: \operatorname{Spec}(R) \rightarrow \mathcal{M}$.

Now let $M$ be an $(A, \Gamma)$-comodule. Define a sheaf $\mathcal{F}_{M}$ on $\mathcal{M}=\mathcal{M}(A, \Gamma)$ by setting

$$
\mathcal{F}_{M}(R, x) \stackrel{\text { def }}{=} \mathcal{F}_{M}(x: \operatorname{Spec}(R) \rightarrow \mathcal{M})=R \otimes_{A} M .
$$


To get the transition functions, note that a 2-commuting diagram

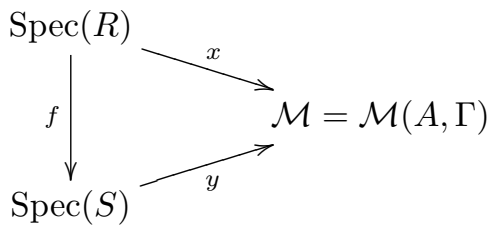

corresponds to a diagram of the form

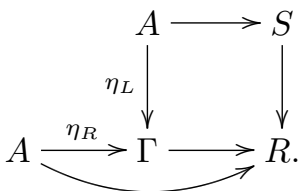

and, in particular, we get a map $\epsilon: S \otimes_{A} \Gamma \rightarrow R$ by push-out. The transition function

$$
\mathcal{F}_{M}(S, y) \longrightarrow \mathcal{F}_{M}(R, x)
$$

is then given by the composition

$$
S \otimes_{A} M \stackrel{S \otimes \psi}{\longrightarrow} S \otimes_{A} \Gamma \otimes_{A} M \stackrel{\epsilon \otimes M}{\longrightarrow} R \otimes_{A} M
$$

Thus from every $(A, \Gamma)$ comodule we get a quasi-coherent sheaf of $\mathcal{O}_{\mathcal{M}}$-modules. ${ }^{6}$ As asserted above, this is actually part of an equivalence of categories between comodules and quasi-coherent sheaves. To get the inverse functor, let $u: \operatorname{Spec}(A) \rightarrow \mathcal{M}$ represent the universal element. Then, we can assign to any quasi-coherent sheaf $\mathcal{F}$ the $A$-module $\mathcal{F}(A, u)$ with comodule structure obtained from the Cech resolution of Example 1.8.

Remark 1.13 (Other topologies). In the algebraic geometry literature, authors focus on some variation of the étale topology. In [27], for example, the standard topology is the "smooth-étale" topology. This is the category of smooth maps $\operatorname{Spec}(R) \rightarrow \mathcal{M}$ with the topology coming from étale maps $\operatorname{Spec}(S) \rightarrow \operatorname{Spec}(R)$. This is not particularly well-suited to the homotopy theoretic applications I have in mind, because smooth and étale have finiteness assumptions that don't apply; for example, the Lazard ring is not finitely generated as a commutative ring over $\mathbb{Z}$. But Mike Hopkins has observed that we can replace smooth and étale by weaker notions that do apply, and which fit well with the obstruction theory of the second half of this note. For example, a morphism $\operatorname{Spec}(R) \rightarrow \mathcal{M}$ could be called quasi-smooth if it is flat, if the natural map

$$
\mathbb{L}_{\mathrm{Spec}(R) / \mathcal{M}} \rightarrow \Omega_{\mathrm{Spec}(R) / \mathcal{M}}
$$

from the cotangent complex to the Kaehler differentials is an isomorphism in homology. Similarly, quasi-étale would mean flat and that there is an equivalence $\mathbb{L}_{\operatorname{Spec}(R) / \mathcal{M}} \simeq 0$. The cotangent complex and its relation to the differentials is explained briefly below in section 2.4. The notion of quasismoothness is very closely related to the notion of formal smoothness as defined by Grothendieck, et al. See [23], §III.3.1. In addition to the stipulations of quasi-smooth, it is required that $\Omega_{\mathrm{Spec}(R) / \mathcal{M}}$ be a projective $R$-module.

\footnotetext{
${ }^{6}$ Recall ([10] or [27],§13) that a sheaf $\mathcal{F}$ of $\mathcal{O}$-modules is quasi-coherent if $\mathcal{F}(R, x)$ is an $R$-module for each $x$ : $\operatorname{Spec}(R) \rightarrow \mathcal{M}$, and if for every 2-commuting diagram $\theta=(f, \phi)$ the induced map of $S$-modules $\theta^{*} \mathcal{F}(R, x) \rightarrow \mathcal{F}(S, y)$ is an isomorphism.
} 
Example 1.14 (Cohomology). Let $M$ be an $(A, \Gamma)$-comodule and $\mathcal{F}_{M}$ the associated quasicoherent sheaf on $\mathcal{M}=\mathcal{M}(A, \Gamma)$. In Example 1.12 we saw that all quasi-coherent sheaves were of this form. Let $\mathcal{U}$ be the $\operatorname{cover} \operatorname{Spec}(A) \rightarrow \mathcal{M}$. Then using Example 1.8 and the previous discussion we immediately get an isomorphism

$$
\check{H}^{*}\left(\mathcal{U}, \mathcal{F}_{M}\right) \cong \operatorname{Ext}_{\Gamma}^{*}(A, M) .
$$

between the Čech cohomology and the comodule Ext groups. We would like to know that this actually computes the cohomology of the stack with coefficients in $\mathcal{F}_{M}$. This can be seen as follows. We have a flat cover $u: \operatorname{Spec}(A) \rightarrow \mathcal{M}$ and this induces adjoint functors

$$
u^{*}: \mathbf{S h} / \mathcal{M} \rightleftarrows \mathbf{S h} / \operatorname{Spec}(A): u_{*}
$$

where I am writing $\mathbf{S h} /(-)$ for quasi-coherent sheaves. Since $u^{*} \mathcal{F}=\mathcal{F}(A, u)$ regarded only as an $A$-module, adjointness implies that if $\tilde{N}$ is the quasi-coherent sheaf over $\operatorname{Spec}(A)$ defined by an $A$ module $N$, then $u_{*} \tilde{N}$ is the quasi-coherent sheaf associated to the extended comodule $\Gamma \otimes_{A} N$. In particular, $u_{*}$ is exact (since $\Gamma$ is flat over $A$ ), and we get

$$
H^{s}\left(\mathcal{M}, q_{*} \tilde{N}\right) \cong H^{s}(\operatorname{Spec}(A), \tilde{N})
$$

for all $s$. Furthermore,

$$
H^{s}(\operatorname{Spec}(A), \tilde{N})=0
$$

if $s>0$. The last uses a comparison of cohomology for the flat topology and the Zariski topology. See, for example, Proposition III.3.7 of [31]. The usual argument (for example, §III.4 of [19]) now gives a natural isomorphism from Cech cohomology to sheaf cohomology and we obtain

$$
H^{*}\left(\mathcal{M}, \mathcal{F}_{M}\right) \cong \operatorname{Ext}_{\Gamma}^{*}(A, M) .
$$

for all quasi-coherent sheaves $\mathcal{F}_{M}$.

Example 1.15 (Invariant differentials). Until now we have been working in an ungraded context, which is not very topological. We now put in the gradings, at least for stacks over the moduli stack of formal group laws. The standard device here is to invoke the sheaf of invariant differentials.

If $G$ is a formal group law over $R$, then the invariant differentials $\omega_{G} \subseteq R[[x]] d x$ are the differentials $f(x) d x$ so that

$$
f\left(x+{ }_{G} y\right) d\left(x+{ }_{G} y\right)=f(x) d x+f(y) d y .
$$

This is a free $R$-module of rank 1 with generator

$$
\frac{d x}{G_{x}(0, x)} .
$$

These invariant differentials assemble into a quasi-coherent sheaf $\omega$ on $\mathcal{M}_{\mathcal{F G} \mathcal{L}}$. The sheaf $\omega$ is invertible and we obtain sheaves $\omega^{\otimes t},-\infty<t<\infty$. Thus, we define the $\mathbb{Z}$-graded structure sheaf

$$
\mathcal{O}_{*}=\left(\mathcal{O}_{\mathcal{F} \mathcal{G L}}\right)_{*}=\left\{\omega^{\otimes t}\right\} .
$$

Topological considerations force $\omega$ itself to be in degree 2; the sheaf $\mathcal{O}_{\mathcal{F G} \mathcal{L}}$ is in degree 0 . If

$$
x: \operatorname{Spec}(R) \rightarrow \mathcal{M}_{\mathcal{F G} \mathcal{L}}
$$


is an element of Flat $/ \mathcal{M}_{\mathcal{F} \mathcal{G} \mathcal{L}}$ and actually represents a formal group law over $R$, then

$$
\mathcal{O}_{*}(R, x) \cong R\left[u^{ \pm 1}\right]
$$

where $u$ is traditionally assigned degree -2 , to be the generator for the tangent space of the formal group determined by the coordinate. As in Example 1.9, this formal group law will be Landweber exact, so there is a 2-periodic homology theory $E$ with $E_{*} \cong R\left[u^{ \pm 1}\right]$.

For a general $x: \operatorname{Spec}(R) \rightarrow \mathcal{M}_{\mathcal{F G} \mathcal{L}}$ of Flat $/ \mathcal{M}_{\mathcal{F} \mathcal{G} \mathcal{L}}$, we have that

$$
\mathcal{O}_{*}(R, x) \cong S_{R}\left(\omega_{x}^{ \pm 1}\right)
$$

is the graded $R$-algebra on the invertible $R$-module $\omega_{x}=\omega(R, x)$.

These ideas extend to stacks over the moduli stack of formal group laws. If $\mathcal{M} \rightarrow \mathcal{M}_{\mathcal{F G} \mathcal{L}}$ is a representable morphism of algebraic stacks, we can pull back $\omega$ to a sheaf on $\mathcal{M}$, and similarly grade the structure sheaf of $\mathcal{M}$.

\subsection{The realization problem}

The question becomes whether and how one might lift the structure sheaf of a stack to homotopy theory. We would hope, at least, for a presheaf of spectra of some sort, and the ambient model category would be the one determined by the topology on Flat $/ \mathcal{N}$ for some algebraic stack $\mathcal{N}$. I am going to use the language and results of [24] and [25]. In particular, any presheaf of spectra $Y$ has an associated homotopy sheaf $\pi_{*} Y$, which is the sheaf associated to the presheaf

$$
(x: \operatorname{Spec}(R) \rightarrow \mathcal{N}) \mapsto \pi_{*} Y(R, x) .
$$

Weak equivalences are morphisms that induce isomorphisms on homotopy sheaves, and cofibrations are "pointwise"; that is,

$$
Y(R, x) \rightarrow Z(R, x)
$$

is a cofibration of the underlying objects (spectra, $E_{\infty}$-ring spectra, etc.) for each object of Flat $/ \mathcal{N}$. The fibrations and fibrant objects are called, respectively, global fibrations and globally fibrant objects. In this way, the topology makes the model category structure. If $Y$ is such a presheaf, then the global sections of a globally fibrant model for $Y$ form the homotopy global sections $(h \Gamma)(Y)$ and, under reasonable hypotheses, there is a descent spectral sequence

$$
H^{s}\left(\mathcal{N}, \pi_{t} Y\right) \Longrightarrow \pi_{t-s}(h \Gamma)(Y) .
$$

One optimistic way to phrase the realization problem is this:

Problem 1.16 (Realization Problem I). Let $\mathcal{N} \rightarrow \mathcal{M}_{\mathcal{F G L}}$ be a representable morphism of algebraic stacks. Is there a presheaf of $E_{\infty}$-ring spectra $X_{\mathcal{N}}$ equipped with an isomorphism of sheaves of commutative rings

$$
\pi_{*} X_{\mathcal{N}} \cong\left(\mathcal{O}_{\mathcal{N}}\right)_{*} ?
$$

I don't think anyone expects this problem, or some of its obvious variations, to have an affirmative answer in all cases; thus a good result would a workable condition on the morphism of stacks $\mathcal{N} \rightarrow \mathcal{M}_{\mathcal{F G} \mathcal{L}}$ which guaranteed a solution.

Let me now explore this problem with a sequence of examples. 
Example 1.17 (The discrete case). The first example would be the case of a morphism $x$ : $\operatorname{Spec}(R) \rightarrow \mathcal{M}_{\mathcal{F} \mathcal{G} \mathcal{L}}$. If, for example, the morphism $x$ is actually represented by a formal group law over $R$, we are asking whether there is a 2-periodic $E_{\infty}$ ring spectrum $X_{R}$ so that $\pi_{*}\left(X_{R}\right)=R\left[u^{ \pm 1}\right]$ and so that the associated formal group law is the one we started with. Certainly $E_{\infty}$ is too much to hope for here, as the case of Morava $K$-theory shows, but we could ask for $A_{\infty}$ instead. Here much is known, and some answers are explored below. (See section 2.5). In the case when $x: \operatorname{Spec}(R) \rightarrow \mathcal{M}_{\mathcal{F G \mathcal { L }}}$ is flat, then Example 1.9 provides a homotopy commutative ring spectrum $E$ with the desired property, and I know of no example which does not lift to an $E_{\infty}$ ring spectrum.

Example 1.18 (Formal group laws). The obvious global example is the case of the identity map of $\mathcal{M}_{\mathcal{F} \mathcal{G} \mathcal{L}}$ to itself; that is, can the graded structure sheaf $\left(\mathcal{O}_{\mathcal{F G} \mathcal{L}}\right)_{*}$ be lifted to a presheaf of $E_{\infty}$ ring spectra? In this form, the problem was raised explicitly by Hopkins and Miller in [8], and has a long history. As Haynes put it, "Morava is back there somewhere" and I gather that Cartier also made some remarks along these lines. This would have the effect of, in some sense, solving the local problem of the previous example, at least for Landweber exact theories, all at once.

If there is such a lifting $X_{\mathcal{F G} \mathcal{L}}$, then the homotopy global sections would be the stable zero-sphere $S^{0}$ and the descent spectral sequence would be the Adams-Novikov spectral sequence. That is, there would be an isomorphism of spectral sequences

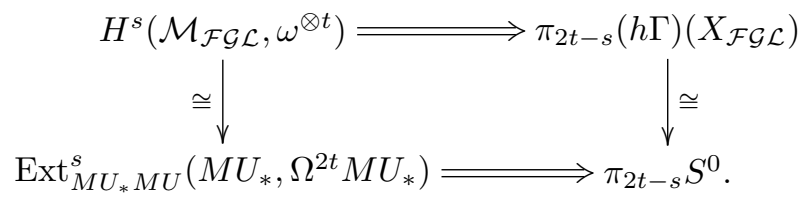

where, for any graded module $M$, with $\left(\Omega^{r} M\right)_{n}=M_{n+r}$. Part of the assertion here is an isomorphism on $E_{2}$ terms. This is known to be true and is a change of rings result; see [34] $\S 15$ for the exact analog of this result in the elliptic case. See also [11] for a similar result in the formal case.

Example 1.19 (Flat maps). We could add the hypothesis that the representable morphism $\mathcal{N} \rightarrow$ $\mathcal{M}_{\mathcal{F G \mathcal { L }}}$ of algebraic stacks be flat. Expanding on Example 1.9, this would have the effect of producing a diagram in the homotopy category of spectra with the desired property. One of the original programs for constructing the spectrum tmf (as in some notes of a course given by Mike Hopkins at MIT) is to apply the techniques of Dwyer and Kan [12] to lift to a diagram of structured spectra. This applies to the case of the morphism

$$
\mathcal{M}_{\text {ell }} \longrightarrow \mathcal{M}_{\mathcal{F} \mathcal{G} \mathcal{L}} .
$$

See Example 1.10. The homotopy global sections of the realization of the structure sheaf of $\mathcal{M}_{\text {ell }}$ would be the spectrum tmf of topological modular forms studied, by Hopkins, Miller, Mahowald, Ando, Strickland, Rezk, and others. The name arises from the fact that the ring of global sections $H^{0}\left(\mathcal{M}_{\text {ell }},\left(\mathcal{O}_{\text {ell }}\right)_{*}\right)$ is the graded ring of modular forms. The question of which modular forms survive to $E_{\infty}$ in the descent spectral sequence has led to some deep and original mathematics. See the notes for Mike Hopkins's 2002 ICM talk [20].

I should say immediately that I am being naïve in this case, because of the subtleties of Example 1.10. However, one should be able to understand a presheaf of structured ring spectra realizing the structure sheaf on $\mathcal{M}_{\text {ell }}^{+}$and tmf would be the connective cover of the homotopy global sections of this presheaf. 
Now let $E_{*}$ be some homology theory with $E_{*} E$ flat over $E_{*}$, so that we get a good theory of comodules. Instead of trying to realize a graded ring as the homotopy groups of a spectrum, we could try to realize an $E_{*}$-algebra in $E_{*} E$-comodules as the $E_{*}$-homology of some ring spectrum. I'd like to restate the realization problem in this guise. I, for one, am more comfortable with this version of the problem.

Rather than use any one homology theory, let's notice that Example 1.9 gives a presheaf

$$
\mathcal{O}_{*}(-)=\left(\mathcal{O}_{\mathcal{F} \mathcal{G} \mathcal{L}}\right)_{*}(-)
$$

of homology theories on $\mathcal{M}_{\mathcal{F} \mathcal{G} \mathcal{L}}$. It's value at $x: \operatorname{Spec}(R) \rightarrow \mathcal{M}_{\mathcal{F} \mathcal{G} \mathcal{L}}$ is, by Landweber exactness,

$$
(\mathcal{O}(R, x))_{*} X=R\left[u^{ \pm 1}\right] \otimes_{M U_{*}} M U_{*}(X)
$$

at least if $x$ is represented by an actual formal group law. More globally, $\mathcal{O}_{*} X$ is the sheaf associated to the graded $(A, W)$ comodule $M U P_{*}(X)$, where $M U P$ is the 2-periodic version of $M U$. See [2] and [39]. This is a sheaf of good homology theories in the sense defined above. The co-operations are given locally (and perhaps after faithfully flat extension) by

$$
R\left[u^{ \pm 1}\right] \otimes_{M U_{*}} M U_{*} M U \otimes_{M U_{*}} R\left[u^{ \pm 1}\right] .
$$

These assemble into a sheaf $\mathcal{O}_{*} \mathcal{O}$ over $\mathcal{M}_{\mathcal{F G}} \mathcal{L}$ which could be intrinsically defined by the formula

$$
\left[\mathcal{O}_{*} \mathcal{O}\right]\left(\operatorname{Spec}(R) \rightarrow \mathcal{M}_{\mathcal{F} \mathcal{G} \mathcal{L}}\right)=\mathcal{O}_{*}\left(\operatorname{Spec}(R) \times_{\mathcal{M}} \operatorname{Spec}(R) \rightarrow \mathcal{M}_{\mathcal{F} \mathcal{G} \mathcal{L}}\right)
$$

The pair $\left(\mathcal{O}_{*}, \mathcal{O}_{*} \mathcal{O}\right)$ forms a sheaf of Hopf algebroids on $\mathcal{M}_{\mathcal{F} \mathcal{G} \mathcal{L}}{ }^{7}$ Note that this construction works for any representable flat morphism $\mathcal{N} \rightarrow \mathcal{M}_{\mathcal{F G}}$ of algebraic stacks and we can ask to what extent this can be realized by a presheaf of ring spectra.

Problem 1.20 (Realization Problem II). Let $\mathcal{N} \rightarrow \mathcal{M}_{\mathcal{F G} \mathcal{L}}$ be a representable flat morphism of algebraic stacks. Is there a presheaf of $E_{\infty}$-ring spectra $X$ on $\mathcal{N}$ equipped with an isomorphism of sheaves of comodule algebras

$$
X_{*} X \cong \mathcal{O}_{*} \mathcal{O} ?
$$

There are multiple reasons for rephrasing the problem in this manner, as I hope to explain in the next section. See also Problem 2.1 below.

\section{Moduli Spaces and Obstruction Theory}

The realization problem I stated at the end of the last section was of the following general nature: can we lift some slice of algebraic geometry to algebraic topology? I would like to develop here an obstruction theory for this problem which has, as its input, inherently algebraic data. In other words, I would like to set up a system where I could use algebraic geometry to start towards a solution.

What I am not going to do is worry about the necessary point-set foundations of spectra, structured ring spectra, pre-sheaves of such, and all the problems that arise there. There are very real issues here, and many people have thought about them. I would simply like to assert that it will work out. Call me Master Pangloss, but these days we always seem to be able to find the best of all possible worlds when we need it. A careful analysis of what is needed and where the necessary technology can be found in the literature can be found in the first section of [18] - at least in the non-presheaf case.

\footnotetext{
${ }^{7}$ But $\mathcal{O}_{*} \mathcal{O}$ is not quasi-coherent as a left $\mathcal{O}_{*}$-module.
} 


\subsection{The Dwyer-Kan moduli space}

Let us begin by choosing a very good homology theory $E_{*}$. At the very least, I mean $E$ is a homotopy commutative ring spectrum, and the $E_{*} E$ is flat over $E_{*}$. At a later point, I will also need that $E$ satisfies the condition spelled out by Adams in [1] §III.13. This condition, which first appeared in a paper by Atiyah [5] is used to build a universal coefficient spectral sequence and is also used by us [17] to build good model category structures on simplicial $E_{*} E$ comodules. Any Landweber exact theory is very good in this sense. See [33].

Here are some variations on the basic realization problem 1.20.

Problem 2.1 (Realization Problem IIbis). Fix a very good homology theory E.

1.) Let $A$ be a (commutative) $E_{*}$-algebra in $E_{*} E$ comodules. Is there an $A_{\infty}$ (or $\left.E_{\infty}\right)$ ring spectrum $X$ so that $E_{*} X \cong A$ ?

2.) More generally, let $k$ be a given $E_{\infty}$-ring spectrum and $A$ a (commutative) $E_{*} k$-algebra in $E_{*} E$-comodules. Is there an $A_{\infty}\left(\right.$ or $\left.E_{\infty}\right) k$-algebra $X$ so that $E_{*} X \cong A$ ?

3.) More globally, let $A$ be a sheaf of $E_{*}$ algebras in $E_{*} E$ comodules where $E_{*}(-)$ is some sheaf of very good homology theories. Is there a presheaf of $A_{\infty}$ or $E_{\infty}$-ring spectra $X$ so that there is an isomorphism of sheaves $E_{*} X \cong A$ ?

Each one of these problems has sub-problems and variants. Under 1.), for example, we could take $A=E_{*} E$ itself. It is this case for which Robinson's original obstruction theory (see [35] and [6]) applied, at least in the $A_{\infty}$ case. More recently ([36]) Robinson has an $E_{\infty}$ version based on Gamma cohomology [37]. For 2.) we might consider a graded formal group law classified by a map of graded rings $M U_{*} \rightarrow R$ and set

$$
A=M U_{*} M U \otimes_{M U_{*}} R
$$

as an $M U_{*} M U$ algebra. See section 2.5. And 3.), of course, is a variant on the realization problem of 1.20; it too has a relative version, as in Problem 2.1.2.

There is a moduli space parametrizing all solutions to such problems, due to Dwyer and Kan [13]. For example, for Problem 2.1.1, let $\mathcal{R}(A)$ be the category with objects all $A_{\infty}$ ring spectra $X$ with $E_{*} X \cong A$ and morphisms $E_{*}$-equivalences of $A_{\infty}$-ring spectra. The category $\mathcal{R}(A)$ is not small, but it is homotopically small in a certain precise sense, and, taking the nerve, we have a weak equivalence of spaces

$$
B \mathcal{R}(A) \simeq \coprod_{[X]} \operatorname{BAut}^{h}(X)
$$

where $X$ runs over representatives for the $E_{*}$-equivalence classes of solutions and $\operatorname{Aut}^{h}(X)$ is the monoid of self-weak equivalences. Here and elsewhere, mapping objects will be taken in the derived sense: take a fibrant/cofibrant replacement for $X$ and then calculate the space of weak equivalences.

It is immediately clear that $B \mathcal{R}(A)$ is non-empty if and only if the problem has a solution and that $\pi_{0} B \mathcal{R}(A)$ parametrizes the different $E_{*}$-equivalence classes of solutions. More than that, as I hope to explain, this gives a place to start making calculations.

It is also immediately clear that this construction and results can be reformulated in the other variants of the realization problem listed in 2.1. In fact, let me now follow the policy of stating 
results and ideas in the case of the basic problem 2.1.1, and (perhaps) pointing to what needs to be changed to cover the more general cases. I now formulate what I consider to be the computable analog of the problems stated so far.

Problem 2.2 (Realization Problem III). For any of the Realization Problems 1.16, 1.20, or 2.1, calculate the homotopy type of the associated Dwyer-Kan moduli space.

\subsection{André-Quillen cohomology}

In an algebraic context, André-Quillen cohomology is always derived functors of derivations. Let me first talk about the classical definitions, then review the changes that are needed when dealing with algebras and modules over them in the category of comodules.

Most basically, if $A$ is an associative $k$-algebra, and $M$ is an $A$-bimodule, then I will write André-Quillen cohomology as

$$
R^{s} \operatorname{Der}_{k}(A, M)=\pi^{s} \operatorname{Der}_{k}(Y, M)
$$

where $\operatorname{Der}_{k}(-, M)$ denotes the $k$-module of derivations and $Y \rightarrow M$ is a cofibrant simplicial resolution of $A$ as a simplicial associative $k$-algebra. Note that if $A$ is commutative and $M$ is an $A$-module, then we could resolve $A$ by a cofibrant simplicial commutative $A$ algebra $Z$ and perform the same construction, but we get a different cohomology theory. The relationship between the two is explained below in section 2.4. The standard (and beautiful) reference for this material is Quillen's paper [32].

This has a reformulation which allows for easy generalization. If $M$ is an $A$-bimodule write $M \rtimes A$ for the module $M \times A$ with $k$-algebra multiplication

$$
(a, x)(b, y)=(a y+x b, x y)
$$

This is the split square-zero extension of $A$ by $M$, and

$$
\operatorname{Der}_{k}(A, M)=\operatorname{Hom}_{k-\operatorname{alg} / A}(A, M \rtimes A) .
$$

The functor $(-) \rtimes A$ extends to simplicial $A$-bimodules. If we let $K(M, n)$ be the simplicial bimodule with normalized chain complex $M$ concentrated in degree $n$, then

$$
\begin{aligned}
R^{s} \operatorname{Der}_{k}(A, M) & \left.\cong \pi_{0} \operatorname{map}_{k-\operatorname{alg} / A}(A, K(M, s) \rtimes A)\right) \\
& \left.\cong \pi_{i} \operatorname{map}_{k-\operatorname{alg} / A}(A, K(M, s+i) \rtimes A)\right)
\end{aligned}
$$

(Here and elsewhere, mapping spaces will be taken to be the derived mapping spaces obtained by tak-

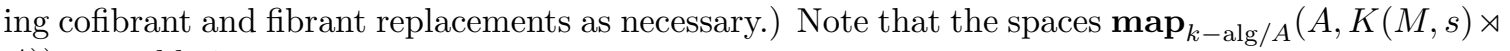
A)) assemble into a spectrum

$$
\operatorname{map}_{k-\operatorname{alg} / A}(A, K(M) \rtimes A)
$$

and we can give a very nice formula

$$
R^{s} \operatorname{Der}_{k}(A, M) \cong \pi_{-s} \operatorname{map}_{k-\operatorname{alg} / A}(A, K(M) \rtimes A) .
$$


In our context, the commutative algebra $k$, the $k$-algebra $A$, and the bimodule $M$ are all $E_{*} E$ comodules and all the multiplications are comodule maps. I'll write

$$
\operatorname{Der}_{E_{*}-k}(A, M)=\operatorname{Hom}_{E_{*}-k-\operatorname{alg}}(A, M \rtimes A)
$$

for the module of derivations in $E_{*} E$ comodules, and I'll write

$$
R^{s} \operatorname{Der}_{E_{*}-k}(A, M)=\pi_{-s} \operatorname{map}_{E_{*}-k-\operatorname{alg}}(A, K(M) \rtimes A)
$$

for the resulting cohomology theory. One of the points of [17] is that there are appropriate model categories so that the derived mapping spaces needed for this construction can be computed there. But as word of warning, unlike the classicial case, not every simplicial algebra in $E_{*} E$ comodules is fibrant, and one must resolve both source and target to interpret the formula of 2.2. This problem is confronted in [23] as well.

Remark 2.3. This is not as abstract as it seems.

1.) In the classical case, $R^{*} \operatorname{Der}_{k}(A, M)$ is essentially a derived variant of Hochschild cohomology. Indeed

$$
R^{s} \operatorname{Der}_{k}(A, M) \cong \operatorname{Ext}_{A \otimes_{k}^{\mathbb{L}} A^{o p}}^{s+1}(A, M), \quad s \geq 1
$$

and there is four-term sequence in low degrees

$$
0 \rightarrow Z(M) \rightarrow M \rightarrow \operatorname{Der}_{k}(A, M) \rightarrow \operatorname{Ext}_{A \otimes_{k}^{\mathbb{L}} A^{o p}}^{1}(A, M) \rightarrow 0
$$

Here we are using a type of hyper-ext over the derived tensor product which degenerates to Ext if $A$ is flat over $k$. I'll explain more in the next paragraph. Also

$$
Z(M)=\{a \in M \mid a x=x a \text { for all } x \in A\} .
$$

It is worth elaborating a bit on the hyper-ext, as it always seems to cause confusion. The derived tensor product $A \otimes_{k}^{\mathbb{L}} A^{o p}$ can be modeled by the simplicial algebra $Y \otimes_{k} Y^{o p}$ where $Y \rightarrow A$ is a cofibrant model for $A$ a simplicial associative $k$-algebra. Then

$$
\begin{aligned}
\operatorname{Ext}_{A \otimes_{k}^{\mathbb{L}} A^{o p}}^{s}(A, M) & \cong \pi_{0} \operatorname{map}_{Y \otimes_{k} Y^{o p}-\operatorname{bimod}}(Y, K(M, s)) \\
& \cong \pi_{-s} \operatorname{map}_{Y \otimes_{k} Y^{o p}-\operatorname{bimod}}(Y, K(M))
\end{aligned}
$$

Since we are working with associative algebras, this has an expression as the homology groups of some derived Hom functor over the differential graded algebra gotten by normalizing $Y \otimes_{k} Y^{o p}$.

2.) In the comodule case, if $M=E_{*} E \otimes_{E_{*}} M_{0}$ is the extended comodule on an $A$-bimodule $M_{0}$, then the comodule theory reduces to the classical theory

$$
R^{s} \operatorname{Der}_{E_{*}-k}(A, M) \cong R^{s} \operatorname{Der}_{k}\left(A, M_{0}\right) .
$$

This is essentially because the extended comodule functor is exact (remember $\Gamma$ is flat over $A$ ) and free algebras in comodules are free algebras. See [17].

3.) This all can be globalized to the case of a sheaf of algebras and a sheaf of modules in some Grothendieck topos. In the classical commutative case, this was done at length in [23], but in all cases, the definition remains the same: simply adapt the formula of 2.2 . 


\subsection{The Dwyer-Kan-Stover decomposition of $B \mathcal{R}(A)$.}

In [7], working out a hint supplied in the paper [14] of Dwyer, Kan, and Stover, we gave a decomposition of the Dwyer-Kan moduli space of a П-algebra. The techniques are extremely general, and apply to give a decomposition of the moduli spaces for our problems.

In order to write out the results in a concise form, let $k$ be a commutative algebra, $A$ a $k$-algebra, and $M$ an $A$-bimodule, all in $E_{*} E$ comodules. Write

$$
\mathcal{H}^{s}(A, M)=\operatorname{map}_{E_{*}-k-\operatorname{alg}}(A, K(M, s) \rtimes A) .
$$

This is the (derived) mapping space; it has the property that

$$
\pi_{i} \mathcal{H}^{s}(A, M)=R^{s-i} \operatorname{Der}_{E_{*}-k}(A, M)
$$

Let $\operatorname{Aut}(A, M)$ be the automorphism group of the pair $(A, M)$; by definition, this is the group of $k$-algebra automorphisms of the split square-zero extension $M \rtimes A$ that preserve the splitting. By naturality, the space $\mathcal{H}^{s}(A, M)$ has an action by group $\operatorname{Aut}(A, M)$. Write $\check{\mathcal{H}}^{s}(A, M)$ for the Borel construction or homotopy orbits of this action:

$$
\check{\mathcal{H}}^{s}(A, M)=E \operatorname{Aut}(A, M) \times \operatorname{Aut}\left(A, M \mathcal{H}^{s}(A, M) .\right.
$$

One obvious bimodule for $A$ is $A$ itself; let us write $\Omega^{t} A$ for the shifted bimodule with $\left(\Omega^{t} A\right)_{n}=A_{n+t}$.

Here's what we get. We can write

$$
B \mathcal{R}(A) \simeq \operatorname{holim}_{n} B \mathcal{R}_{n}(A)
$$

where

$$
B \mathcal{R}_{0}(A)=B_{A^{*}}-k-\operatorname{alg}(A)
$$

and for all $n \geq 1$ there is a homotopy pull-back diagram

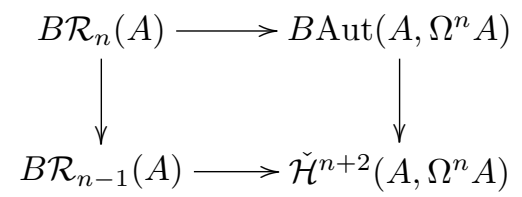

This decomposition has some immediate consequences. The space $B \mathcal{R}_{1}(A)$ is non-empty and path-connected. In attempting to lift a vertex of this space up the tower to $B \mathcal{R}(A)$ we encounter successively defined obstructions in

$$
\pi_{0} \check{\mathcal{H}}^{n+2}\left(A, \Omega^{n} A\right) \cong R^{n+2} \operatorname{Der}_{E_{*}-k-\operatorname{alg}}\left(A, \Omega^{n} A\right) / \operatorname{Aut}\left(A, \Omega^{n} A\right), \quad n \geq 1
$$

Thus the obstruction is an equivalence class of elements in the "(-2)-stem"

$$
R^{n+2} \operatorname{Der}_{E_{*}-k-\operatorname{alg}}\left(A, \Omega^{n} A\right) .
$$

This equivalence class must be that of 0 (which is fixed by $\operatorname{Aut}(A, M)$ ) in order to go on.

Next, the fiber at a vertex in $B \mathcal{R}_{n-1}(A)$ is either empty or the space $\mathcal{H}^{n+1}\left(A, \Omega^{n} A\right)$. Thus the "(-1)-stem" holds the obstructions to unique lifting. Furthermore, given a vertex of $B \mathcal{R}(A)-$ that 
is, an actual realization $X$ of $A$ - this tower and the decomposition of the Dwyer-Kan moduli space 2.1 gives a spectral sequence for computing $\pi_{*} B \mathcal{R}(A)$. In fact, reindexing the usual spectral sequence of a tower of fibrations gives a second quadrant spectral sequence abutting to $\pi_{t-s+1} B \mathcal{R}(A)$ with

$$
\begin{aligned}
& E_{2}^{0,0}=\pi_{1} B \mathcal{R}_{0}(A) \cong \operatorname{Aut}_{E_{*}-k-\operatorname{alg}}(A) \\
& E_{2}^{s, t}=\pi_{t-s+1} \mathcal{H}^{t+1}\left(A, \Omega^{t} A\right) \cong R^{s} \operatorname{Der}_{E_{*}-k-\operatorname{alg}}\left(A, \Omega^{t} A\right), \quad(s, t) \neq(0,0) .
\end{aligned}
$$

Since derivations often arise in, for example, the Bousfield-Kan spectral sequence for a mapping space, the form of this spectral sequence should not be a surprise. ${ }^{8}$

Finally, the methods are so general that we can extend these results to the other problems of 2.1 , suitably modifying what we mean by the cohomology spaces $\mathcal{H}\left(A, \Omega^{n} A\right)$, of course.

\subsection{Commutative vs non-commutative: the role of the cotangent complex}

In his original paper on the subject [32], Quillen showed how to relate the cohomology of a commutative algebra $A$, regarded as an associative algebra, to the commutative algebra homology of $A$. Let's review that here.

If $A$ is an associative $k$-algebra, let $I_{A / k}$ denote the kernel of the multiplication map $A \otimes_{k} A \rightarrow A$. This is an $A$-bimodule and it represents derivations: if $M$ is an $A$-bimodule, there is a natural isomorphism

$$
\operatorname{Der}_{k}(A, M) \cong \operatorname{Hom}_{A^{e}}\left(I_{A / k}, M\right) .
$$

Here $A^{e}=A \otimes_{k} A^{o p}$, so that the category of $A$-bimodules is equivalent to the category of left $A^{e}$-modules. ${ }^{9}$ The functor $I_{A / k}$ can be derived: let

$$
\mathbb{L} I_{A / k}=A^{e} \otimes_{Y e} I_{Y / k}
$$

where $Y$ is any cofibrant resolution of $A$ as a $k$-algebra. Let us regard $\mathbb{L} I_{A / k}$ as both a simplicial $A^{e}$-module and a chain complex of $A^{e}$-modules, as is convenient. Put another way, I will use the Dold-Kan theorem to confuse $\mathbb{L} I_{A / k}$ with its normalization.

This allows us to rewrite cohomology as follows. Let $C_{*} R$ denote the category of $\mathbb{Z}$-graded chain complexes of left $R$-modules. This has an internal Hom functor $\operatorname{hom}_{R}$ and

$$
R^{s} \operatorname{Der}_{k}(A, M)=H_{-s} \operatorname{hom}_{A^{e}}\left(\mathbb{L} I_{A / k}, M\right) .
$$

This formula is true as written in the classical case. In the comodule case, we have to suitably derive hom in the category of $A^{e}$-modules in $E_{*} E$-comodules. In practice this means taking some sort of injective (or flasque) resolution of $M$ and regarding it as a negatively graded chain complex. The topos case (as in [23]) requires a similar construction.

If $A$ is a commutative $k$-algebra, then we can form the module $\Omega_{A / K}=I_{A / k} /\left(I_{A / k}\right)^{2}$ of Kaehler differentials. If $M$ is an $A$-module, then

$$
\operatorname{Der}_{k}(A, M) \cong \operatorname{Hom}_{A}\left(\Omega_{A / k}, M\right) .
$$

\footnotetext{
${ }^{8}$ We could ask for the Dwyer-Kan-Stover decomposition to arise as the standard decomposition of some cosimplicial space. I know of no such result.

${ }^{9}$ The $e$ is for enveloping algebra.
} 
This can derived: let

$$
\mathbb{L}_{A / k}=A \otimes_{Z} \Omega_{Z / k}
$$

where $Z \rightarrow A$ is a cofibrant resolution of $A$ as a simplicial commutative $k$-algebra. This is the cotangent complex of $A$ as a $k$-algebra; its homology is the André-Quillen homology of $A$ as a commutative $k$-algebra. Again, regard $\mathbb{L}_{A / k}$ as both a simplicial object and a chain complex, as is convenient.

Combining Equation 2.6 with Equation 2.8 immediately implies that

$$
\Omega_{A / k}=A \otimes_{A^{e}} I_{A / k} .
$$

Also, using Equation 2.7 we have for $A$ commutative and $M$ an $A$-module

$$
R^{s} \operatorname{Der}_{k}(A, M)=H_{-s} \operatorname{hom}_{A}\left(A \otimes_{A^{e}} \mathbb{L} I_{A / k}, M\right) .
$$

Then, using a suitable bicomplex, we get Quillen's commutative to non-commutative spectral sequence:

$$
H^{p} \operatorname{hom}_{A}\left(\Lambda_{q+1} \mathbb{L}_{A / k}, M\right) \Longrightarrow R^{p+q} \operatorname{Der}_{k}(A, M) .
$$

Here I have written $H^{p}$ hom(-) for $H_{-p}$ hom(-) to get the first quadrant nature of the spectral sequence correct, and $\Lambda_{q} \mathbb{L}_{A / k}$ denotes the $q$ th exterior power of the cotangent complex. ${ }^{10}$

This easily globalizes to the sheaf setting. As in [23], the objects $\mathbb{L} I_{A / k}, \mathbb{L}_{A / k}$, and $\Lambda^{q} \mathbb{L}_{A / k}$ are inherently local, and yield sheaves. Then in the spectral sequence 2.9 we use the appropriate notion of a global hom functor.

\section{$2.5 \quad M U$-algebras}

As a test case for the preceding technology, I would like to discuss certain types of $M U$-algebras. There is an extensive literature for the kind of examples I am going to give below. See [15] and [40] for homotopy theoretic results, and [6], [35] and (especially) the work of Lazarev [28] for more rigid $A_{\infty}$ results. Jeff Smith has also thought extensively about such things.

In this section I will write $\mathbb{L}(A / k)$ (instead of $\left.\mathbb{L}_{A / k}\right)$ for the cotangent complex of a $k$-algebra $A$ because I will have some complicated arguments for this bifunctor.

Definition 2.4. Suppose we have a morphism of $q: M U_{*} \rightarrow A$ of $\mathbb{Z}$-graded commutative rings; then $A$ is an $M U_{*}$-algebra. We define $A$ to be a chromatic $M U_{*}$-algebra if

1.) If $J$ is the kernel of $q$, then $J$ is generated, as an ideal, by a regular sequence;

2.) the cotangent complex $\mathbb{L}\left(A /\left(M U_{*} / J\right)\right)$ is contractible.

The class of chromatic $M U_{*}$ algebras is closed under localization, étale extension, and filtered colimits; therefore, many of the familiar examples of spectra under $M U$ provide examples of chromatic algebras. This list includes, for example,

i.) $B P_{*}=\mathbb{Z}_{(p)}\left[v_{1}, v_{2}, \ldots\right]$;

ii.) $B P\langle n\rangle_{*}=\mathbb{Z}_{(p)}\left[v_{1}, v_{2}, \ldots, v_{n}\right]$;

\footnotetext{
${ }^{10}$ Which is inherently derived, as $\mathbb{L}_{A / k}$ is a cofibrant simplicial $A$-module.
} 
iii.) $E(n)_{*}=\mathbb{Z}_{(p)}\left[v_{1}, \ldots, v_{n-1}, v_{n}^{ \pm 1}\right]$;

iv.) $K(n)_{*}=\mathbb{F}_{p}\left[v_{n}^{ \pm 1}\right]$;

v.) $P(n)_{*}=\mathbb{F}_{p}\left[v_{n}, v_{n+1}, v_{n+2}, \ldots\right]$

and many of the two-periodic analogs of these algebras. One example might be the algebra obtained from $E(n)_{*}$ by adjoining a $\left(p^{n}-1\right)$ st root of $v_{n}$; this is the $M U_{*}$ algebra

$$
\mathbb{Z}_{(p)}\left[u_{1}, u_{2}, \ldots, u_{n-1}\right]\left[u^{ \pm 1}\right]
$$

where the degree of $u_{i}$ is zero and the degree of $u$ is -2 . The localization of this algebra at the maximal ideal $\left(p, u_{1}, \ldots, u_{n-1}\right)$ remains chromatic.

While the algebra homomorphism $M U_{*} \rightarrow A$ determines a graded formal group law, the property of being chromatic is not an invariant under strict isomorphisms of formal group laws. Indeed, over $\mathbb{Q} \otimes M U_{*}$ the universal formal group law is isomorphic to the additive formal group law and the former is chromatic, but the latter is not. In this sense, this section is a bit tangential to the rest of the paper.

A chromatic algebra $A$ is necessarily concentrated in even degrees; if not, the cotangent complex of Definition 2.4.2 could not be contractible. Therefore, it follows immediately from the AtiyahHirzebruch spectral sequence that if $A$ can be realized by a spectrum $X$, we must have

$$
M U_{*} X \cong M U_{*} M U \otimes_{M U_{*}} A \stackrel{\text { def }}{=} M U_{*} A
$$

as an $M U_{*} M U$ algebra in $M U_{*} M U$ comodules. Thus, after applying the appropriate analog of Remark 2.3.2, the homotopy type of the Dwyer-Kan moduli spaces of $A$ by associative (that is, $A_{\infty}$ with $M U$ in the center) $M U$-algebras is governed by the André-Quillen cohomology groups

$$
R^{s} \operatorname{Der}_{M U_{*} M U}\left(M U_{*} A, \Omega^{t} A\right) .
$$

Theorem 2.5. Let $A$ be a chromatic $M U_{*}$-algebra. Then

1.) $R^{s} \operatorname{Der}_{M U_{*} M U}\left(M U_{*} A, \Omega^{t} A\right)=0$ unless $t$ is even and $t-s$ is odd.

2.) There is an isomorphism

$$
\mathbb{L}\left(M U_{*} A / M U_{*} M U\right) \simeq \Sigma\left(M U_{*} A \otimes_{M U_{*} / J} J / J^{2}\right)
$$

in the derived category of $M U_{*} A$-modules. In particular, $H_{*} \mathbb{L}\left(M U_{*} A / M U_{*} M U\right)$ is concentrated in degree $q=1$ and is a free $M U_{*} A$ module.

This result is actually more concrete that I'm letting on; it is possible to give a complete calculation of $R^{s} \operatorname{Der}_{M U_{*} M U}\left(M U_{*} A, \Omega^{t} A\right)$. Part 2.) of this result is a routine exercise with properties of the cotangent complex that go back to André and Quillen: the main ingredients are flat base change, the transitivity sequence, and the contractibility hypothesis I've built into the definition of a chromatic algebra. Part 1.) can be proved using the spectral sequence of Equation 2.9 or directly using Remark 2.3.1.

To apply the commutative to non-commutative spectral sequence, the key calculation is that there is an isomorphism in the derived category of $M U_{*} A$ modules

$$
\Lambda^{q} \mathbb{L}\left(M U_{*} A / M U_{*} M U\right) \simeq \Sigma^{q} \Gamma^{q}\left(M U_{*} A \otimes_{M U_{*} / J} J / J^{2}\right)
$$


where $\Gamma^{q}$ is the $q$ th homogeneous piece of the divided power algebra functor. This is a free $M U_{*} A$ module.

To see the isomorphism of 2.11 , regard $\mathbb{L}\left(M U_{*} A / M U_{*} M U\right)$ as a simplicial $M U_{*} A$-module and let $\Lambda$ denote the exterior algebra functor. Then Theorem 2.5.2 implies

$$
\pi_{*} \Lambda\left(\mathbb{L}\left(M U_{*} A / M U_{*} M U\right)\right) \cong \Gamma\left(M U_{*} A \otimes_{M U_{*} / J} J / J^{2}\right)
$$

with

$$
M U_{*} A \otimes_{M U_{*} / J} J / J^{2} \cong \pi_{1} \Lambda\left(\mathbb{L}\left(M U_{*} A / M U_{*} M U\right)\right)
$$

and $\Gamma(-)$ the divided power algebra functor. The needed isomorphism follows by looking at homotopy in degree $q$.

An immediate corollary of this calculation is the following. Note that on a traditional Adams spectral sequence chart, where the axes are $(t-s, s)$, the non-zero groups of Theorem 2.5.1 appear in odd columns and odd rows - a kind of modified "checkerboard" pattern. ${ }^{11}$ Thus we immediately have that the obstruction groups 2.5 vanish and the spectral sequence at the end of section 2.3 collapses, except possibly for differentials beginning with

$$
E_{2}^{0,0}=\operatorname{Aut}_{M U_{*} M U-\operatorname{alg}}\left(M U_{*} A\right) .
$$

In the examples above, this group is actually trivial or, in the case of 2.10 , finite. In any case, we the following result.

Theorem 2.6. Let $A$ be a chromatic algebra, then there exists an $M U$-algebra (in the rigid $A_{\infty}$ sense) so that $M U_{*} X \cong M U_{*} A$ as an $M U_{*} M U$ algebra.

As the groups $R^{s} \operatorname{Der}_{M U_{*} M U}\left(M U_{*} A, \Omega^{t} A\right)$ with $t-s=-1$ are anything but trivial, there may be many (in fact, uncountably many) such structures. This phenomenon is familiar. See [35].

\subsection{Hopkins-Miller solves a moduli problem}

The Hopkins-Miller theorem is sometimes summarized as saying that there is an $A_{\infty}$ or $E_{\infty}$ structure on the Morava $E$-spectrum (or Lubin-Tate spectrum) $E_{n}$. If this was all we really wanted, the result is essentially due to Baker [6] in its $A_{\infty}$ form, or one could follow Robinson [36] for the $E_{\infty}$ case. $^{12}$ But Hopkins and Miller did much more. Among other things, they showed that the space of $A_{\infty}$ self maps of $E_{n}$ is contractible and calculated the components, thereby getting an action of the Morava stabilizer group on $E_{n}$. But even this statement still doesn't give the complete picture. I would like to explain how they solved a moduli problem of the sort I've been discussing here. The reference in print is [33]. See also [18].

The first point is that it is even a bit of misstatement to speak of the Morava $E$-theory at all. Fix a prime $p$ and an integer $n \geq 1$. Define a category $\mathcal{H} t_{n}$ as follows. The objects are morphisms

$$
x: \operatorname{Spec}(k) \rightarrow \mathcal{M}_{\mathcal{F G} \mathcal{L}}
$$

where $k$ is an algebraic extension of the prime field $\mathbb{F}_{p}$ and the resulting formal group law has height $n$. The morphisms are the 2-commuting triangles. Note that the prime is suppressed from the notation here and throughout.

\footnotetext{
${ }^{11}$ I'm told a similar pattern appears in the elliptic case.

${ }^{12}$ But Robinson needs some version of Theorem 2.8 below, which is due to Hopkins and Miller.
} 
Let $\operatorname{Alg}_{c}$ denote the category of complete local $\mathbb{Z}_{p}$ algebras, where $\mathbb{Z}_{p}$ denotes the $p$-adic integers. If $x: \operatorname{Spec}(k) \rightarrow \mathcal{M}_{\mathcal{F} \mathcal{G} \mathcal{L}}$ is actually given by a formal group law $G$ over $k$, then Lubin-Tate theory [29] yields an object $R(k, x) \in \mathbf{A l g}_{c}$ and a formal group law $H$ over $R(k, x)$ so that the pair $(R(k, x), H)$ represents $\star$-isomorphism classes of deformations of $G$. As a ring

$$
R(k, x) \cong W(k)\left[\left[u_{1}, \cdots, u_{n-1}\right]\right]
$$

where $W(k)$ is the ring of Witt vectors over $k$. The formal group law $H$ over $R(k, x)$ is unique only up to $\star$-isomorphism; however, for any choice of $H$, the classifying map $L \rightarrow R(k, x)$ is Landweber exact and we get a homology theory $E(k, x)_{*}$ with $E(k, x)_{0} \cong R(k, x)$. Any one of these might be called Morava $E$-theory.

The assignment $(k, x) \mapsto R(k, x)$ is a functor of the pair $(k, x)$; hence faithfully flat descent yields a functor

$$
\mathcal{E}: \mathcal{H} t_{n}^{o p} \longrightarrow \mathbf{A l g}_{c} \text {. }
$$

As in Problem 1.20 we can form the diagram $\left(\mathcal{E}_{*}, \mathcal{E}_{*} \mathcal{E}\right)$ of Hopf algebroids. Let $B \mathcal{R}\left(\mathcal{E}_{*} \mathcal{E}\right)$ be the Dwyer-Kan moduli space (in $A_{\infty}$ rings spectra) for this diagram.

Theorem 2.7 (Hopkins-Miller). There is a unique (up to weak equivalence) diagram

$$
E: \mathcal{H} t_{n}^{o p} \longrightarrow A_{\infty} \text { ring spectra }
$$

equipped with an isomorphism of diagrams of Hopf algebroids

$$
\left(E_{*}, E_{*} E\right) \cong\left(\mathcal{E}_{*}, \mathcal{E}_{*} \mathcal{E}\right) .
$$

Indeed, there is a weak equivalence

$$
B \mathcal{R}\left(\mathcal{E}_{*} \mathcal{E}\right) \simeq K\left(\mathbb{Z}_{p}^{\times} \times \widehat{\mathbb{Z}}, 1\right)
$$

where $\widehat{\mathbb{Z}}$ is the profinite completion of the integers.

We can replace $A_{\infty}$ by $E_{\infty}$. See [18]. The essential ingredient of the proof of this result or any result like it is that for any map $x: \operatorname{Spec}(k) \rightarrow \mathcal{M}_{\mathcal{F G} \mathcal{L}}$ in $\mathcal{H} t_{n}$, the unit map

$$
k \rightarrow k \otimes_{E(k, x)_{0}} E(k, x)_{0} E(k, x)
$$

is quasi-étale - that is, it is flat and has vanishing cotangent complex. It is, in fact, a colimit of étale maps. One way to record this for input into the technology I've outlined here is the next result. Let $\mathcal{O}_{*}: \mathcal{H} t_{n}^{o p} \rightarrow$ Rings be the "graded structure sheaf"; thus, at $x: \operatorname{Spec}(k) \rightarrow \mathcal{M}_{\mathcal{F G} \mathcal{L}}$

$$
\mathcal{O}_{*}(k, x) \cong k\left[u^{ \pm 1}\right] .
$$

This is the coefficient ring of the associated Morava $K$-theory.

Theorem 2.8. There is an isomorphism in the derived category of $\mathcal{O}_{*} \otimes_{\mathcal{E}_{*}} \mathcal{E}_{*} \mathcal{E}$ modules

$$
\mathcal{O}_{*} \otimes_{\mathcal{E}_{*}} \mathbb{L}_{\mathcal{E}_{*} \mathcal{E} / \mathcal{E}} \simeq 0
$$


This result, the discussion at the end of section 2.3 , the fact that $\mathcal{E}_{*}$ is a diagram of complete local rings, and the spectral sequence 2.9 are enough to imply that $B \mathcal{R}\left(\mathcal{E}_{*} \mathcal{E}\right)$ is weakly equivalent to the classifying space of the automorphism group of the diagram of Hopf algebroids $\left(\mathcal{E}_{*}, \mathcal{E}_{*} \mathcal{E}\right)$. Thus, to finish the discussion, I need to tell you a little about this automorphism group.

By the results of $[33] \S 17$, this automorphism group is isomorphic to to the group of natural transformations of the forgetful functor

$$
F: \mathcal{H} t_{n} \rightarrow \text { Schemes } / \mathcal{M}_{\mathcal{F G} \mathcal{L}}
$$

Note that every endomorphism of this functor is an isomorphism. Restricting such a natural transformation $\phi$ to any

$$
x: \operatorname{Spec}(k) \rightarrow \mathcal{M}_{\mathcal{F} \mathcal{G} \mathcal{L}}
$$

defines a homomorphism of our automorphism group to the center of

$$
S_{n}(k, x) \rtimes \operatorname{Gal}\left(k / \mathbb{F}_{p}\right)
$$

where $S_{n}(k, x)$ is the automorphism group of the formal group law - often called the Morava stabilizer group of the formal group law over $k$. If $i: \operatorname{Spec}(L) \rightarrow \operatorname{Spec}(k)$ is given by an extension of fields, then

$$
S_{n}(k, x) \rightarrow S_{n}\left(L, i^{*} x\right)
$$

is an injection. Also, all height $n$ formal group laws over the algebraically closed field $\overline{\mathbb{F}}_{p}$ are isomorphic. Combining these two facts yields an injection from our automorphism group to the center of

$$
S_{n}\left(\overline{\mathbb{F}}_{p}, x\right) \rtimes \operatorname{Gal}\left(\overline{\mathbb{F}}_{p} / \mathbb{F}_{p}\right)
$$

for any map $x: \operatorname{Spec}\left(\overline{\mathbb{F}}_{p}\right) \rightarrow \mathcal{M}_{\mathcal{F G} \mathcal{L}}$ in $\mathcal{H} t_{n}$.

The center of the group 2.12 is $\mathbb{Z}_{p}^{\times} \times \operatorname{Gal}\left(\overline{\mathbb{F}}_{p} / \mathbb{F}_{p}\right)$, but not every element arises as the image of a natural transformation. The elements of $\mathbb{Z}_{p}^{\times}$are exactly the $n$-series $[n](x)$ with $n \in \mathbb{Z}_{p}^{\times}$; these certainly give rise to natural transformations. Then Proposition 21.5 of [33] (and its evident converse) imply that the automorphism group is isomorphic to

$$
\mathbb{Z}_{p}^{\times} \times \sigma^{n} \operatorname{Gal}\left(\overline{\mathbb{F}}_{p} / \mathbb{F}_{p}\right)
$$

where $\sigma$ is the Frobenius and

$$
\sigma^{n} \operatorname{Gal}\left(\overline{\mathbb{F}}_{p} / \mathbb{F}_{p}\right) \subset \operatorname{Gal}\left(\overline{\mathbb{F}}_{p} / \mathbb{F}_{p}\right)
$$

is the subgroup generated by the $n$th power of the Frobenius. Since $\operatorname{Gal}\left(\overline{\mathbb{F}}_{p} / \mathbb{F}_{p}\right) \cong \widehat{\mathbb{Z}}$, Theorem 2.7 follows.

\section{References}

[1] Adams, J. F., Stable homotopy and generalised homology, Chicago Lectures in Mathematics, University of Chicago Press, Chicago, Ill., 1974.

[2] Ando, Matthew, "Power operations in elliptic cohomology and representations of loop groups", Trans. Amer. Math. Soc., 352 (2000) No. 12, 5619-5666. 
[3] Ando, M. and Hopkins, M. and Strickland, N., "The sigma orientation is an $H_{\infty}$ map", manuscript, UIUC, 2002.

[4] Artin, M., "Versal deformations and algebraic stacks", Invent. Math., 27 (1974), 165-189.

[5] Atiyah, M. F., "Vector bundles and the Künneth formula", Topology, 1 (1962), 245-248.

[6] Baker, Andrew, " $A_{\infty}$ structures on some spectra related to Morava $K$-theories", Quart. J. Math. Oxford Ser. (2), 42 (1991), No. 168, 403-419.

[7] Blanc, David, and Dwyer, William and Goerss, Paul, "The realization space of a П-algebra, manuscript, to appear in Topology.

[8] Carlsson, Gunnar, "Problem session", Equivariant stable homotopy theory and related areas (Stanford, CA, 2000), Homology Homotopy Appl., 3 (2001) No. 2, vii-xv (electronic).

[9] Deligne, P. and Mumford, D.J, "The irreducibility of the space of curves of given genus", Inst. Hautes Études Sci. Publ. Math., 36, (1969), 75-109

[10] Demazure, Michel and Gabriel, Pierre, Groupes algébriques. Tome I: Géométrie algébrique, généralités, groupes commutatifs, Avec un appendice Corps de classes local par Michiel Hazewinkel, Masson \& Cie, Éditeur, Paris, 1970.

[11] Devinatz, Ethan S., "Morava's change of rings theorem", The Čech centennial (Boston, MA, 1993), Contemp. Math. 181, 83-118, Amer. Math. Soc., Providence, RI, 1985.

[12] Dwyer, W. G. and Kan, D. M., "An obstruction theory for diagrams of simplicial sets", Nederl. Akad. Wetensch. Indag. Math., 46 (1984) No. 2, 139-146.

[13] Dwyer, W. G. and Kan, D. M., "A classification theorem for diagrams of simplicial sets", Topology, 23 (1984), No. 2, 139-155.

[14] Dwyer, W. G. and Kan, D. M. and Stover, C. R., "The bigraded homotopy groups $\pi_{i, j} X$ of a pointed simplicial space X", J. Pure Appl. Algebra, 103 (1995) No. 2, 167-188.

[15] Elmendorff, A.D., Kriz, I., Mandell, M.A., May, J.P., "Rings, modules, and algebras in stable homotopy theory", Mathematical Surveys and Monographs 47, AMS, Providence, RI, 1996.

[16] Fantechi, Barbara, "Stacks for everybody", European Congress of Mathematics, Vol. I (Barcelona, 2000), Progr. Math., 201, 249-359, Birkhäuser, Basel, 2001.

[17] Goerss, Paul G. and Hopkins, Michael J., "André-Quillen (co)-homology for simplicial algebras over simplicial operads, Une dégustation topologique: homotopy theory in the Swiss Alps (Arolla, 1999), Contemp. Math. 265, 41-85, Amer. Math. Soc., Providence, RI 2000.

[18] Goerss, Paul G. and Hopkins, Michael J., "Moduli spaces of commutative ring spectra", preprint, Northwestern Univerity, 2003.

[19] Hartshorne, Robin, Algebraic geometry, Graduate Texts in Mathematics, No. 52, SpringerVerlag, New York, 1977. 
[20] Hopkins, M.J., "Algebraic topology and modular forms", manuscript MIT, 2003. Submitted to the Proceedings of the ICM, Beijing, 2002.

[21] Hopkins, M. J. and Gross, B. H., "The rigid analytic period mapping, Lubin-Tate space, and stable homotopy theory", Bull. Amer. Math. Soc. (N.S.), 30 (1994) No. 1, 76-86.

[22] Hollander, S. "A Homotopy Theory for Stacks", Thesis, MIT 2001.

[23] Illusie, Luc, Complexe cotangent et déformations. I, Lecture Notes in Mathematics, Vol. 239, Springer-Verlag, Berlin, 1971.

[24] Jardine, J. F., "Stable homotopy theory of simplicial presheaves", Canad. J. Math., 39 (1987) No. 3, 733-747.

[25] Jardine, J. F., "Presheaves of symmetric spectra", J. Pure Appl. Algebra, 150 (2000) No. 2, $137-154$.

[26] Jardine, J. F., "Stacks and the homotopy theory of simplicial sheaves", Equivariant stable homotopy theory and related areas (Stanford, CA, 2000), Homology Homotopy Appl., 3 (2001) No. 2, 361-384.

[27] Laumon, Gérard and Moret-Bailly, Laurent, Champs algébriques, Ergebnisse der Mathematik und ihrer Grenzgebiete. 3. Folge. A Series of Modern Surveys in Mathematics 39, SpringerVerlag, Berlin 2000.

[28] Lazarev, A., "Towers of MU-algebras and the generalized Hopkins-Miller theorem", preprint 2002.

[29] Lubin, Jonathan and Tate, John, "Formal moduli for one-parameter formal Lie groups", Bull. Soc. Math. France, 94 (1966), 49-59.

[30] Miller, Haynes, "A new take on LEFT", notes of a talk given at the AMS Annual Meeting, Baltimore 2003. Available at

http://www-math.mit.edu/ hrm/papers

[31] Milne, James S., Étale cohomology, Princeton Mathematical Series, 33, Princeton University Press, Princeton, N.J., 1980.

[32] Quillen, Daniel, "On the (co-) homology of commutative rings", Applications of Categorical Algebra, Proc. Sympos. Pure Math., Vol. XVII, New York, 1968, 65-87, Amer. Math. Soc., Providence, R.I., 1970.

[33] Rezk, Charles, "Notes on the Hopkins-Miller theorem", Homotopy theory via algebraic geometry and group representations (Evanston, IL, 1997), Contemp. Math. 220, 313-366, Amer. Math. Soc., Providence, RI, 1998.

[34] Rezk, Charles, "Supplementary Notes for Math 512, Version 0.17", available at http://www. math.uiuc.edu/ rezk/. 
[35] Robinson, Alan, "Obstruction theory and the strict associativity of Morava K-theories", $A d$ vances in homotopy theory (Cortona, 1988), London Math. Soc. Lecture Note Ser. 139, 143-152, Cambridge Univ. Press, Cambridge, 1989.

[36] Robinson, Alan, "Gamma homology, Lie representations and $E_{\infty}$ multiplications", Invent. Math. 152 (2003), No.2, 331-348.

[37] Robinson, Alan and Whitehouse, Sarah, "Operads and $\Gamma$-homology of commutative rings", Math. Proc. Cambridge Philos. Soc., 132 (2002) No. 2, 197-234.

[38] Strickland, Neil P., "Finite subgroups of formal groups", J. Pure Appl. Algebra, 121 (1997) No. $2,161-208$.

[39] Strickland, Neil P., "Formal schemes and formal groups", Homotopy invariant algebraic structures (Baltimore, MD, 1998), Cont. Math 239, 263-352, Amer. Math. Soc., Providence, RI, 1999.

[40] Strickland, N. P., "Products on MU-modules", Trans. Amer. Math. Soc., 351 (1999) No. 7, 2569-2606.

[41] Vistoli, Angelo, "Intersection theory on algebraic stacks and on their moduli spaces", Invent. Math., 97 (1989) No. 3, 613-670. 